

Revista Nacional de

ISSN $2318-8472$

v. 05, n. 30,2017

Gerenciamento de Cidades

\title{
Planos e projetos urbanos de campi universitários na cidade contemporânea.
}

Plans and urban projects of university campi in the contemporary city.

Planes y proyectos urbanos para los campus universitarios de la ciudad contemporánea.

Elaine Saraiva Calderari

Arquiteta e Urbanista na Universidade Federal de Uberlândia (UFU) e Doutoranda de Arquitetura e Urbanismo na Universidade de São Paulo (USP)

Elainesc.ufu@gmail.com 


\section{RESUMO}

Esse trabalho investiga as dimensões de atuação da universidade (campus universitário) na sociedade (cidade) e sua interface com um novo agente norteador da conformação espacial no espaço urbano contemporâneo, o mercado, apontando problematizações e tendências do tema, estabelecendo reflexões sobre o papel do campus universitário (como também da instituição pública) e suas possíveis relações com a cidade, a sociedade e os espaços públicos e de domínio público.

Busca-se estabelecer uma leitura apontando casos e experiências capazes de exemplificar as problematizações e tendências dos novos posicionamentos das universidades, principalmente com ênfase nas estratégias de implantação e inserção de suas estruturas físicas e, consequentemente, em suas relações e conexões/articulações com a cidade e a sociedade na contemporaneidade.

PALAVRAS-CHAVE: Campus universitário. Cidade Contemporânea. Espaços universitários.

\section{ABSTRACT}

This work investigates the dimensions of university action in the society (city) and its interface with a new agent of the spatial conformation in the contemporary urban space, the market, pointing out problematizations and tendencies of the theme, establishing reflections on the role of the University campus (as well as the public institution) and its possible relations with the city, society and public spaces and public domain.

It seeks to establish a reading pointing to cases and experiences capable of exemplifying the problematizations and tendencies of the new positions of the universities, mainly with emphasis on the strategies of implantation and insertion of their physical structures and, consequently, in their relations and connections / articulations with the city And society in the contemporary world.

KEY WORDS: University campus. Contemporary city. University spaces

\section{RESUMEN}

Este trabajo investiga las dimensiones de actuación universitaria (campus universitario) en la sociedad (ciudad) y su interfaz con un nuevo agente rector de la conformación espacial en el espacio urbano contemporáneo, el mercado, apuntando problematizaciones y tendencias temáticas, establecer reflexiones sobre el papel de campus universitario (así como la institución pública) y sus posibles vínculos con la ciudad, la sociedad y los espacios de dominio público y privado.

Busca establecer una lectura señalando casos y experiencias que ejemplifican las problematizaciones y tendencias de nuevas posiciones en las universidades, en especial, con énfasis en las estrategias de implementación e integración de sus estructuras físicas y, en consecuencia, en sus relaciones y conexiones / juntas con la ciudad y la sociedad hoy en día.

PALABRAS CLAVE: Campus universitario. Ciudad contemporánea. Espacios universitarios. 


\section{INTRODUÇÃO}

A gênese das universidades passa pela busca da cidade ideal, já que historicamente também se buscava uma universidade ideal, principalmente baseada no ideário do que deveria ser uma universidade, seja na aplicação de princípios norteadores ou nas concepções projetuais, funcionando como uma importante ferramenta para garantir o desenvolvimento e a chamada modernização de um país.

Desta forma, os espaços universitários (assim como as novas cidades planejadas, ditas ideais) acabaram se tornando grandes objetos experimentais de conceitos, ideologias e contradições, sob as mais variadas concepções projetuais de diversos arquitetos e urbanistas em suas distintas épocas, que transcreveram seu imaginário e suas conviç̧ões referentes à formação de modelos urbano-arquitetônicos, "de forma única e não plural, em uma metáfora da modernidade do campus universitário ideal para a cidade" (RODRIGUES, 1997, p. 13-22). E é importante destacar que quando tal fato não foi o suficiente, resolveram criar suas próprias cidades, ditas universitárias.

Historicamente é possível identificar 3 períodos distintos de formação e estruturação do espaço universitário, na tentativa de estabelecer protótipos ou modelos de espaços universitários, sendo o primeiro no contexto da Idade Média, exclusivamente na Europa, com os exemplos de Bolonha (1088), Paris (1150), Oxford (1167), Cambrigde (1209) e Coimbra (1536); o segundo período durante a 1 Guerra Mundial, no contexto Europa e Estados Unidos, com os exemplos de Virginia (1819), Berkeley (1868) e Paris (CIUP, 1920) e o terceiro período durante a 2 Guerra Mundial sob o contexto da América Latina, como os casos do Chile (1931); Venezuela (1943); México (1950); Brasil - UFRJ (1920) e Brasil-UNB (1960).

No primeiro período é possível observar que o surgimento da universidade não apresentava nenhuma relação física com o território. Apenas a partir do momento em que suas atividades são expandidas e ocorre a sua materialização em espaços físicos é que de fato a universidade passa a existir como um equipamento urbano. Observa-se que nesse momento histórico é iniciada uma relação entre a universidade e a escala urbana.

Nota-se uma relação muito clara com a dimensão social e cultural da cidade, com a promoção de continuidades físicas e/ou comportamentais, de forma que a universidade se inseria na malha urbana existente, como também suas atividades eram entendidas como complementares à vida urbana. Apenas com a inserção do modelo inglês, dos chamados colleges, é possível verificar a concentração de atividades em espaços físicos específicos, dando início a um processo de separação física entre atividades cotidianas e atividades universitárias em espaços definidos e limitados.

A influência e o poder da Igreja, até estão soberanos nesse equipamento, passam a ser vislumbrados pelo Estado em busca da dominação do conhecimento, mas também da dominação ideológica. Desta forma, esse equipamento passa a se estabelecer como uma instituição pública e de prestígio na sociedade e, portanto, a inserção em escala monumental 
torna-se importante para que a universidade se transforme em uma instituição relevante dentro da cidade.

No segundo período, além da concentração de atividades, ocorre uma relação de contiguidade entre as estruturas físicas, com a ideia de formação de um espaço único e principalmente distante das atividades cotidianas, independente e autossuficiente, com o isolamento completo das atividades universitárias.

Esse é um momento marcado por uma ruptura ideológica, com o domínio da ciência no ensino (não mais religioso) e na pesquisa, indicando o rompimento completo com a Igreja. Além disso, há a inserção da escala espacial e bucólica por meio do distanciamento físico, marcado pelo seu posicionamento no território dissociado da cidade, com leis e normas próprias, como também a aproximação do espaço rural, representado pela "natureza" como garantia de qualidade de vida e de ensino, com uma suposta preocupação com a dimensão ambiental (não como se vê hoje, mas com a necessidade de proximidade e conexão com os elementos da natureza, o que traria qualidade de vida).

Esse processo promoveu o rompimento com o espaço social, ou seja, com as atividades de sociabilização na cidade, promovendo uma segregação física e social, que encontra seu auge na formulação do conceito de cidades universitárias.

O terceiro período é referente aos contextos latino-americanos, com a aplicação do modelo modernista iniciado nos Estados Unidos, representando a síntese e a experimentação do urbanismo moderno, com a transferência de concepções/conceitos que surgiram para a concretização de uma cidade ideal (funcional), aplicados e concentrados nas chamadas cidades uinversitárias.

A arquitetura e o urbanismo se transformam na materialização de símbolos e na criação de identidades nacionais, em que as instalações universitárias passam a ser vistas como a consolidação das bases políticas do Estado, o que se justifica pela necessidade de modernização de cada país. Além disso, a ascensão da universidade nesses países envolve a necessidade de formação de um quadro com técnicos e profissionais qualificados para seu crescimento e desenvolvimento.

E o que se observa ao se analisar a composição de cada projeto são repetições e analogias de conceitos gerais em todos os exemplos citados, com a formação de uma estrutura autossuficiente e extremamente funcional. Essa estrutura, baseada no uso de eixos de pedestres e veículos, ora separados, ora compartilhados, estabelece o traçado urbano e é rodeada por edifficações setorizadas (zoneamento) em áreas de conhecimento.

Define-se um eixo central que passa a receber um elemento de socialização dos usuários, em grande parte dos exemplos em composição com uma área livre, denominada de praça, onde são posicionados os equipamentos coletivos como biblioteca, reitoria, entre outros.

odo o complexo é pensado como uma estrutura autossuficiente claramente visível, com as poucas referências e/ou indicações de conexões e articulações externas, em sua grande 
maioria estando afastado do centro urbano, como novos polos de crescimento e desenvolvimento da cidade.

Todavia, diferentemente dos períodos mencionados, atualmente a implantação de novos campi na cidade contemporânea enfrenta questionamentos, reflexões e desafios, por estarem estes inseridos nos tecidos urbanos das cidades contemporâneas e compartilharem os efeitos e aspectos das novas formas de produção da cidade e dos novos agentes da conformação espacial dos territórios, principalmente no que se refere à atuação do mercado sob as ações políticas.

\section{A UNIVERSIDADE E A CIDADE CONTEMPORÂNEA}

De acordo com Silva, se, na sua origem, a universidade estava à sombra das tensões e contradições da Igreja e do Estado, atualmente "temos que falar do mercado, e não da Universidade à sombra do mercado, mas de uma Universidade inserida no mercado." (SILVA, 2005, p. 90).

Para o autor, o mercado domina não apenas a universidade, mas aspectos das relações humanas que envolvem sua inserção no espaço urbano. Ele aponta que, nos países europeus, esse processo pode ser mais contido e ainda vem sofrendo certa resistência. Mas, no caso dos países periféricos, como a questão social, de fato, ainda apresenta dificuldades em ser assumida, passa por um acelerado processo de submissão ao mercado, em que a educação passa a ser vista também como um bem de consumo. Silva afirma que "a explicitação dessa inserção varia conforme a tradição da universidade de cada pais, e assim também o ritmo em que o mercado incorpora a instituição universitária, que está ainda em função do ritmo em que absorve todas as outras instituições, devorando o espaço público." (SILVA, 2005, p. 90).

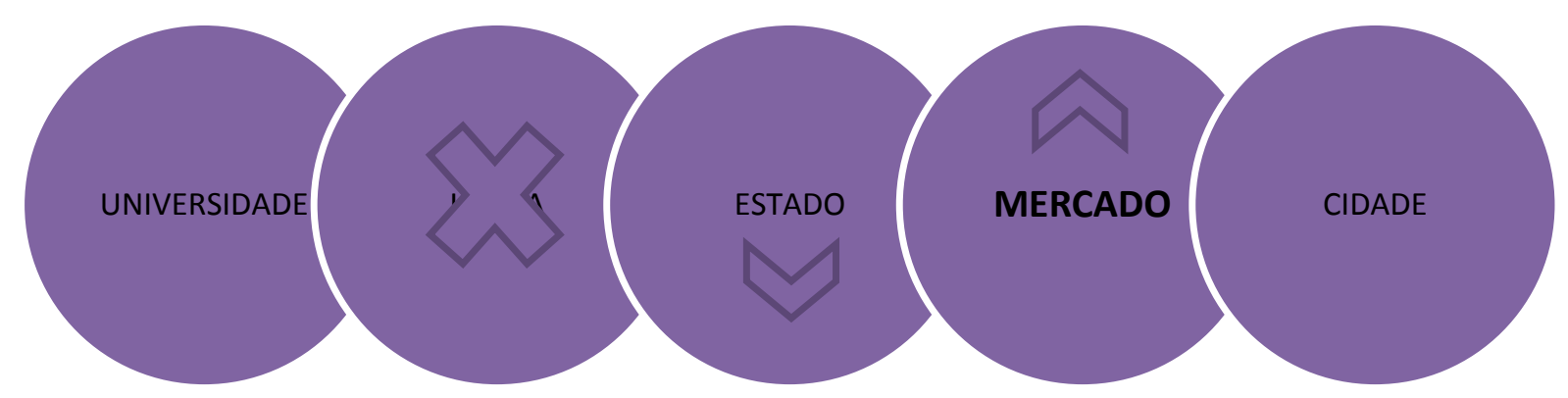

FIGURA 01 | A UNIVERSIDADE E A CIDADE: O AUMENTO DE INFLUÊNCIA DO MERCADO.

Fonte: Autora.

Do mesmo modo, a implantação de uma universidade em qualquer cidade promove impactos sociais, culturais e principalmente econômicos significativos, sendo esta considerada um "atrativo" desejado por todo o governo e a população. Para Nascimento Júnior (2006, p. 163), 


\begin{abstract}
A instalação das [Instituições de Ensino Superior - IES] tende a proporcionar impactos sociais e econômicos significativos aos lugares que the recebem ao possibilitar a geração de emprego, o aumento da arrecadação do município, a dinamização da economia local, etc. (BOVO, 2003). Assim, a fixação desse recurso local pode ser considerada como uma estratégia do poder local vistas as possibilidades criadas com a presença dessas instituições.
\end{abstract}

Todavia, sua implantação também promove significativas transformações urbanas com a penetração do capital em novas esferas de atividade, estimulando a "modernização (tecnológica) e maior 'integração' do território" principalmente utilizando esse equipamento como âncora para a expansão territorial (AMORIN, 2014, p. 5; SOARES, 2013).

Essa expansão, associada à "criação de novos desejos e novas necessidades", promove novas relações e "ampliações nos padrões do consumo" e, ainda, "mudanças nos hábitos dos consumidores", com o surgimento de "novos produtos" urbanos (AMORIN, 2014, p. 4).

Desta forma a implantação dos campi universitários passa a ser também indutora de novos vetores de crescimento e desenvolvimento, sendo rodeada por "novos produtos", como loteamentos fechamentos, shoppings centers, centros de negócios e outros produtos exclusivamente privados, acelerando os processos de segregação físico-espacial já presentes em sua própria conformação espacial (fechada e isolada), transformando-se em uma verdadeira "ilha urbana".

Além disso, quando não ocorre a inserção direta de novos produtos, a valorização da terra de forma exagerada, seja pelo seu valor ou pelo dos imóveis e aluguéis no entorno, já é um forte indício de alteração nas dinâmicas existentes, isso quando não ocorrem processos de gentrificação, com a movimentação da população para lugares cada vez mais distantes (até mais que o próprio campus).

Essa situação se agrava quando a universidade utiliza seus próprios espaços, que deveriam ser públicos, para a inserção desses "novos produtos", passando por processos de privatização "maquiados" de parcerias público-privadas ou de concessões.

\title{
CAMPI UNIVERSITÁRIOS NA CIDADE CONTEMPORÂNEA
}

Desta forma, apenas como uma breve exemplificação da dissociação entre teoria e prática, analisaremos a situação atual das primeiras universidades que adotaram esse modelo (origem) de implantação.

Tanto a Universidade de Virginia como a de Berkeley que deram origem ao modelo de campus universitário com a aplicação dos conceitos de distanciamento e isolamento físico e a promoção de espaços universitários baseados nos princípios de autossuficiência, apresentam duas realidades bem diferentes daquelas defendidas e projetadas em seus planos originais, em 
que a ideologia pretendida/defendida foi completamente alterada ao longo de sua trajetória urbana.

Na Universidade de Virginia, por exemplo, destaca-se uma contradição, quando se verifica a preocupação de promover o isolamento e o distanciamento físico da cidade durante todo o seu processo de gênese. Entretanto, com o processo de crescimento e expansão urbana, não apenas da Universidade, mas da própria cidade, a ideologia inicial não conseguiu conter suas demandas ao longo dos anos, principalmente com o aumento de alunos e de novas estruturas. Assim, o espaço desejado, distante do centro urbano, encontra-se hoje pulverizado na malha urbana da zona oeste da cidade de Charlottesville, conforme identificado na Figura 02, sendo composto por 11 escolas e diversas faculdades, indo muito além do espaço físico destinado ao campus original no "Central Grounds".

Atualmente, é considerada a terceira melhor universidade pública do país, ou seja, seu posicionamento geográfico não foi determinante na qualidade de ensino (assim como sua necessidade de isolamento e concentração em sua origem). Ela é composta por uma população universitária do tamanho de uma cidade de pequeno porte, com mais de 15.600 alunos de graduação e 6.316 de pós-graduação, como também por 14.967 funcionários, sendo que mais de 12.000 são contratados para atividades em tempo integral (UNIVERSITY OF VIRGINIA,2015).

Desta forma, a Universidade de Virginia é considerada como um importante equipamento para o desenvolvimento da cidade, principalmente em relação às questões econômicas, sendo essencial para o funcionamento desta, o que é contraditório quando se observa que, em sua origem, ela pretendia justamente ser independente da cidade.

A|

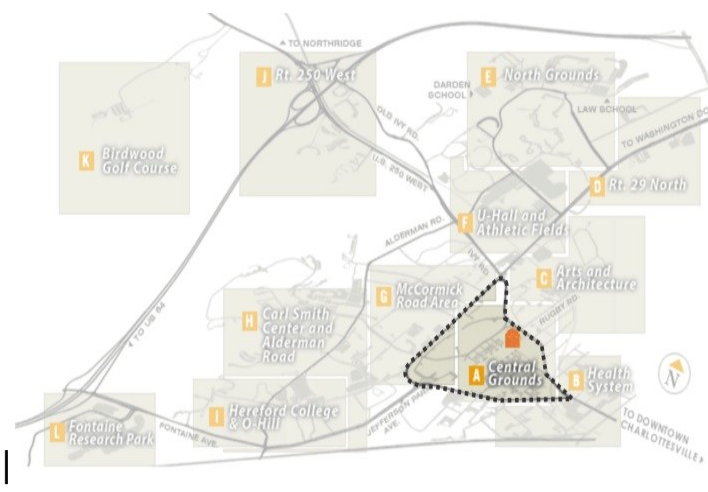

FIGURA 02 | A - MAPA ATUAL DAS ESTRUTURAS DA UNIVERSIDADE DE VIRGINIA EM CHARLOTTESVILLE (DESTAQUE PARA O NÚCLEO PIONEIRO) E B - CAMPUS ORIGINAL(NÚCLEO PIONEIRO) E EXPANSÕES EM SEU ENTORNO IMEDIATO - UNIVERSIDADE DE VIRGINIA.

Fonte: University of Virginia (2016a, 2016b).



\section{(n)} . 


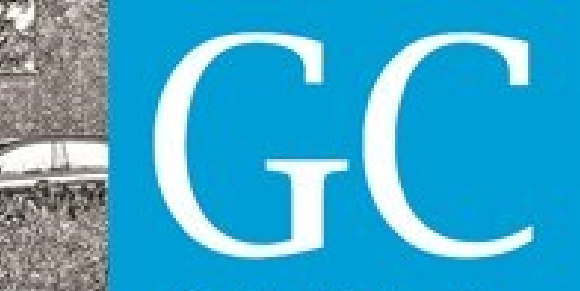

Revista Nacional de

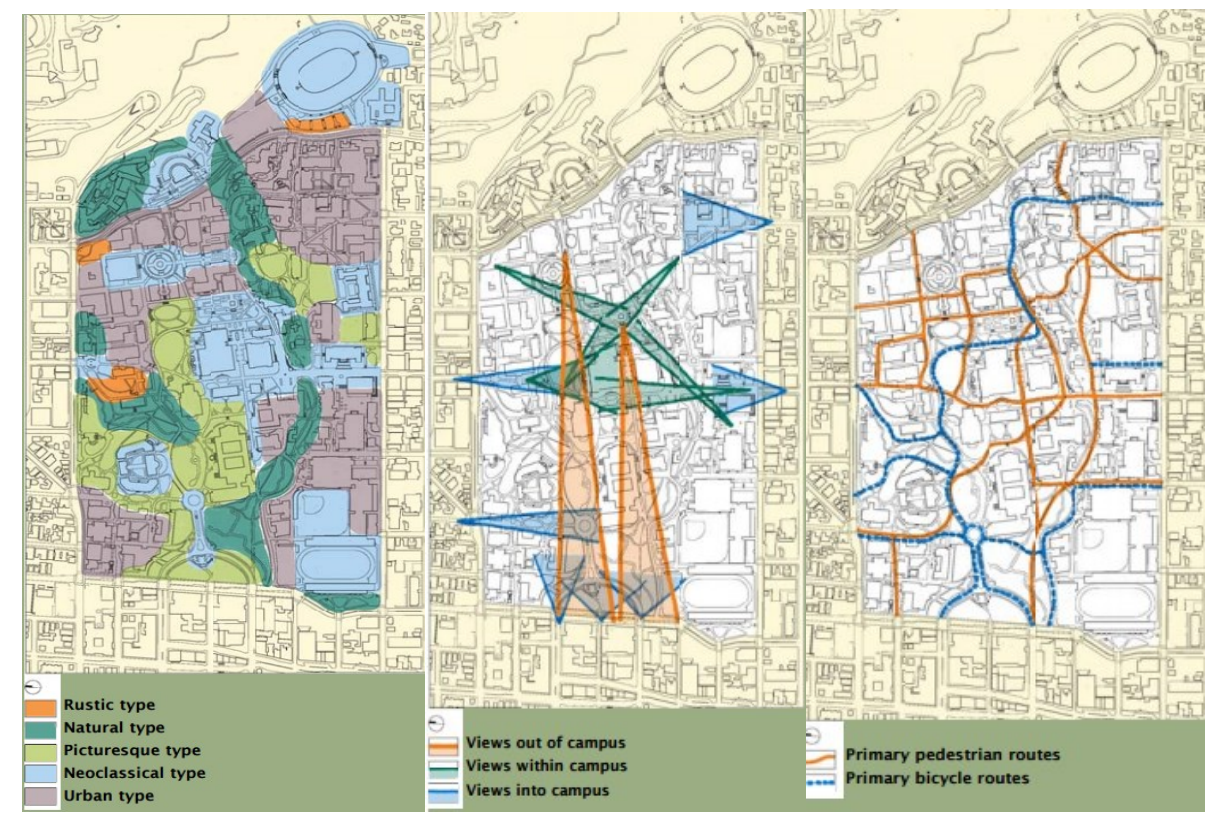

FIGURA 05 | MAPAS TEMÁTICOS DO PLANO DA PAISAGEM DA UNIVERSIDADE DE BERKELEY- CALIFÓRNIA (1868).

Fonte: University of California (2004, p. 10, 13-14, 16).

Atualmente, a Universidade passa pela constatação de uma das consequências da concentração de suas estruturas físicas em espaços únicos e contíguos, o que promove problemas significativos relacionados à oferta de habitação no entorno de seus campi (em virtude da alta valorização da terra e da escassez de preços razoáveis) e aos serviços gerais, como creches, comércios, entre outros, para o atendimento da comunidade universitária. É preciso ressaltar que nem mesmo o Plano da Paisagem conseguiu mitigar essa situação com a falta da população e, portanto, da vida urbana no campus.

Diante dessa situação, a Universidade instituiu um Plano de Desenvolvimento com estratégias e metas a serem cumpridas até 2020 , considerando a questão habitacional como fator-chave em suas decisões, com a estruturação do entorno e a criação de novos campi. Além disso, considerou como fato relevante a necessidade do aumento de ofertas de habitação na região, com meta de crescimento em torno de 32\% (UNIVERSITY OF CALIFORNIA, 2005, p. 25-27).

O Plano de Desenvolvimento 2020 da University of California (2005, p. 25), desenvolvido como uma ação direta da Universidade para a construção de cenários futuros e com a pretensão de solucionar essa questão, determina que

o problema da habitação é particularmente agudo para os estudantes, sendo que a expansão e a melhoria na oferta de habitação perto do campus é fundamental não só para garantir que nossos alunos estejam adequadamente alojados, mas também 
para fornecer estrutura à comunidade dos seus familiares e mentores, como também o acesso aos recursos existentes ${ }^{1}$ no próprio campus".

O Plano de Desenvolvimento 2020 estabeleceu como principais estratégias a busca de parcerias com empreendedores do setor privado na promoção de edificações habitacionais de valores acessíveis e medidas para o controle dos valores dos aluguéis, tentando estabelecer uma reflexão sobre a necessidade de mudanças nas condições do mercado e sensibilizando a população sobre a importância da habitação estudantil para o desenvolvimento não apenas da Universidade, como da própria cidade (UNIVERSITY OF CALIFORNIA, 2005, p. 25-27).

Além disso, propõe parcerias com os órgãos públicos para a criação de políticas públicas que ofereçam a opção de direito de aquisição direta de terrenos e edificações no entorno como prioridade da Universidade, sendo apresentado um zoneamento residencial destacado na cor azul na Figura 06-A, com a delimitação das áreas de interesse habitacional da Universidade, considerando as compatibilidades referentes às normativas de uso e ocupação da cidade e atividades já estabelecidas no local, como comércios, prestações de serviço, creches, etc. (UNIVERSITY OF CALIFORNIA, 2005, p. 25).

Também é proposto um zoneamento, conforme indicado na Figura 06-B, para a localização de novas atividades no campus, sendo destinadas à área do Campus Park apenas as atividades acadêmicas. Já as demais atividades de investigação, com o envolvimento e a participação substancial dos estudantes, reresentadas por museus, recreação, lazer, etc., devem ser pulverizadas no tecido urbano do entorno, como uma forma de integração e interação com a população local (representadas pelas cores verde, roxo e bege). 1 Como exemplo, as áreas de convívio, lazer, recreação, culturais, entre outras, determinadas pelo Plano da
Paisagem. 
É importante destacar que, recentemente, em 2014, a Universidade da Califórnia, em Berkeley, também lançou um novo campus, fato inédito nessa universidade, já que suas expansões se limitavam ao seu entorno imediato. O campus em Richmond (UNIVERSITY OF CALIFORNIA, 2014b), na Califórnia, foi pensado para o desenvolvimento de facilidades adicionais relacionadas com a investigação e a pesquisa, tanto para a UC Berkeley quanto para a Lawrence Berkeley National Laboratory (LBNL).

O campus em Richmond se apresenta como uma tentativa de descentralização de atividades da Universidade para outros espaços físicos e não mais contíguos ao campus inicial. Na Figura 08, é possível observar que todo o plano também foi concebido para a construção de núcleos (indicados nos círculos destacados) com todas as atividades necessárias ao atendimento dos estudantes e da população a serem acrescentados ao campus apenas com o esgotamento das possibilidades previstas em cada núcleo, para então se fazer a expansão de outro núcleo.

De acordo com informação do Plano Diretor do campus, essa decisão buscou evitar construções isoladas e sim como núcleos de convivência, além da otimização de infraestrutura e de edificações eda densificação de usos e atividades de forma concentrada, para estimular a presença e a utilização dos usuários.

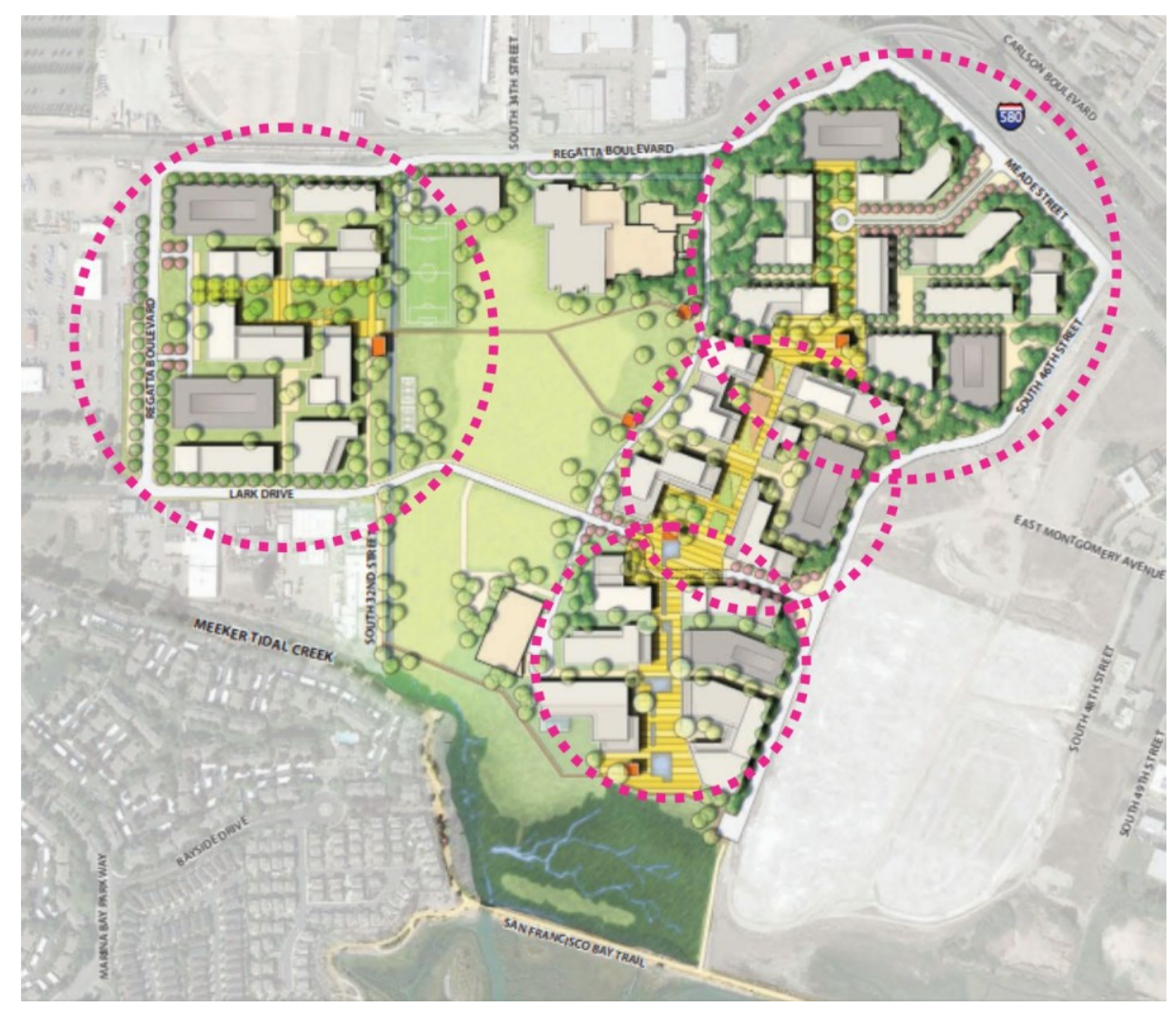

FIGURA 08 | PLANO DE OCUPAÇÃO DO CAMPUS EM RICHMOND - UNIVERSIDADE DE BERKELEY- CALIFÓRNIA (2014). 
Fonte: Adaptado de University of California (2014b, p. 4.11).

\section{CONSIDERAÇÕES FINAIS}

O trabalho buscou uma análise das interfaces das Universidades com a cidade contemporânea por meio dos exemplos utilizados que identificam a situação atual dos modelos-gênese que posteriormente foi aplicado em grande parte dos campi universitários, principalmente no contexto da América Latina, baseado na reprodução dos princípios do urbanismo moderno, das doutrinas do CIAM"2 e do planejamento norte-americano. (SEGAWA, 1998, p. 46).

Tendo em vista tal contexto, este trabalhou apresentou questões relativas às novas espacialidades e territorialidades urbanas como dinâmicas que relacionam transformações econômicas, sociais, ambientais e culturais como práticas no espaço urbano, gerando novas formas de produção da cidade e processos que promovam mudanças no comportamento e na apropriação dos espaços, como os aspectos relacionados aos temas de segregação, homogeneização, fragmentação, transfuncionalidades e hibridação nos campi universitários para a cidade e a sociedade.

Acredita-se que a sobreposição das dimensões socioespaciais seja capaz de estabelecer parâmetros e variáveis de análise para os novos campi universitários que estruturam as tendências e problematizações do espaço urbano contemporâneo, assim como o entendimento das categorias a serem consideradas como potencialidades capazes de promoverem mudanças e transformações significativas na cidade contemporânea quando consideradas como princípios na elaboração tanto do planejamento como de estruturação do projeto urbano.

\section{REFERÊNCIAS BIBLIOGRÁFICAS}

ALVES, M. R. Cidade(s): novas espacialidades e territorialidades. Pós, São Paulo, v. 17, n. 28, p. 250-257, 2010.

ALVES, M. R. Notas de disciplina: IAU5868 - Espaço, Lugar e Espacialidades da Cidade Contemporânea. São Carlos: [s.n.], 2014.

AMORIN, Edna Maria Jucá Couto. O consumo e a reestruturação das cidades médias brasileiras: perspectivas de estudos comparativos. In: SEMINÁRIO INTERNACIONAL RII, 13., 2014, Salvador. Anais... Salvador: [s.n.], 2014. p. 4-5.

BUFFA, E.; PINTO, G. D. A. Arquitetura, urbanismo e educação: campi universitários brasileiros. [S.I.]: [s.n.], 2009.

NASCIMENTO JUNIOR, Francisco das Chagas do. O fenômeno de expansão das instituições de ensino superior e o território brasileiro. Revista Geografia, Londrina, v. 15, n. 1, p. 145-171, jan./jun. 2006.

RODRIGUES, L. A. F. Universidade e a fantasia moderna: a falácia de um modelo espacial único. Niterói: Universidade Federal Fluminense, 1997. 2 CIAM: Congressos Internacionais da Arquitetura Moderna. Foram realizadas 10 edições, com os mais variados
temas. 
SEGAWA, Hugo. Rio de Janeiro, México, Caracas: cidades universitárias e modernidades 1936-1962. Revista de Urbanismo e Arquitetura, Salvador, p. 38-47, 1999. Disponivel em: <http://www.portalseer.ufba.br/index.php/rua/article/viewFile/3133/2249>. Acesso em: 10 set. 2015.

SILVA, F. L. Universidade, cidade, cidadania. In: LANNA, A. L. Cidades universitárias: patrimônio urbanístico e arquitetônico da USP. São Paulo: Ed. USP, 2005.

SOARES, B. Palestra apresentada na Universidade Federal de Uberlândia, Seminário UFU em Expansão. Monte Carmelo: Universidade Federal de Uberlândia, 2013.

UNIVERSITY OF CALIFORNIA (Berkeley). 2014a. Disponível em <http://www.youniversitytv.com/wpcontent/uploads/2014/10/University-of-California-Berkeley-1024x681.jpg>. Acesso em: 10 dez. 2015.

UNIVERSITY OF CALIFORNIA (Berkeley). 2020 Long Range Development Plan. Berkeley, 2005. Disponível em: <http://realestate.berkeley.edu/sites/default/files//rdp_2020.pdf>. Acesso em: 10 jan. 2016.

UNIVERSITY OF CALIFORNIA (Berkeley). Campus architecture. 2002. Disponível em: <http://archives.ced.berkeley.edu/campus-architecture>. Acesso em: 10 dez. 2015.

UNIVERSITY OF CALIFORNIA (Berkeley). By the numbers. Disponível em: <http://www.berkeley.edu/about/bythenumbers>. Acesso em: 20 nov. 2015.

UNIVERSITY OF CALIFORNIA (Berkeley). Landscape Master Plan. Berkeley, Jan. 2004. Disponível em: <http://realestate.berkeley.edu/landscape-master-plan>. Acesso em: 10 jan. 2016.

UNIVERSITY OF CALIFORNIA (Berkeley). Richmond Bay Campus: Long Range Development Plan. Berkeley, May 2014b. Disponível em <http://www.youniversitytv.com/wp-content/uploads/2014/10/University-of-CaliforniaBerkeley-1024x681.jpg>. Acesso em: $10 \mathrm{dez} .2015$.

UNIVERSITY OF VIRGINIA. Facts and Figures. Disponível em: <http://www.virginia.edu/facts>. Acesso em: 20 nov. 2015.

UNIVERSITY OF VIRGINIA. Web Map. Disponível em <http://www.virginia.edu/webmap/>. Acesso em: $10 \mathrm{dez}$. 2016a.

UNIVERSITY OF VIRGINIA. Web Map. Central Grounds. Disponível em <http://www.virginia.edu/webmap/ACentralGrounds.html>. Acesso em: 10 dez. 2016b. 